\title{
Heterologous expression of the atypical tetracycline chelocardin reveals the full set of genes required for its biosynthesis
}

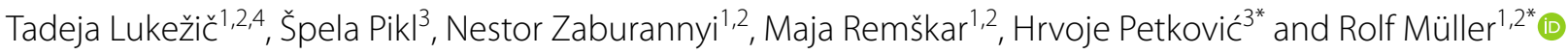

\begin{abstract}
Background: Chelocardin (CHD) exhibits a broad-spectrum antibiotic activity and showed promising results in a small phase II clinical study conducted on patients with urinary tract infections. Importantly, CHD was shown to be active also against tetracycline-resistant Gram-negative pathogens, which is gaining even more importance in today's antibiotic crisis. We have demonstrated that modifications of CHD through genetic engineering of its producer, the actinomycete Amycolatopsis sulphurea, are not only possible but yielded even more potent antibiotics than CHD itself, like 2-carboxamido-2-deacetyl-chelocardin (CD-CHD), which is currently in preclinical evaluation. A. sulphurea is difficult to genetically manipulate and therefore manipulation of the chd biosynthetic gene cluster in a genetically amenable heterologous host would be of high importance for further drug-discovery efforts.
\end{abstract}

Results: We report heterologous expression of the CHD biosynthetic gene cluster in the model organism Streptomyces albus del14 strain. Unexpectedly, we found that the originally defined CHD gene cluster fails to provide all genes required for $\mathrm{CHD}$ formation, including an additional cyclase and two regulatory genes. Overexpression of the putative pathway-specific streptomyces antibiotic regulatory protein chdB in A. sulphurea resulted in an increase of both, CHD and CD-CHD production. Applying a metabolic-engineering approach, it was also possible to generate the potent CHD analogue, CD-CHD in S. albus. Finally, an additional yield increase was achieved in S. albus del 14 by in-trans overexpression of the chdR exporter gene, which provides resistance to CHD and CDCHD.

Conclusions: We identified previously unknown genes in the CHD cluster, which were shown to be essential for chelocardin biosynthesis by expression of the full biosynthetic gene cluster in S. albus as heterologous host. When comparing to oxytetracycline biosynthesis, we observed that the CHD gene cluster contains additional enzymes not found in gene clusters encoding the biosynthesis of typical tetracyclines (such as oxytetracycline). This finding probably explains the different chemistries and modes of action, which make CHD/CD-CHD valuable lead structures for clinical candidates. Even though the CHD genes are derived from a rare actinomycete $A$. sulphurea, the yield of CHD in the heterologous host was very good. The corrected nucleotide sequence of the CHD gene cluster now contains all

\footnotetext{
${ }^{*}$ Correspondence: hrvoje.petkovic@bf.uni-lj.si; rolf.mueller@helmholtz-hips.

de

${ }^{1}$ Department of Microbial Natural Products, Helmholtz Institute for Pharmaceutical Research Saarland (HIPS)-Helmholtz Centre for Infection Research(HZI), and Department of Pharmacy, Saarland University Campus, Campus E8.1, 66123 Saarbrücken, Germany

${ }^{3}$ Department of Food Science and Technology, Biotechnical Faculty,

University of Ljubljana, Jamnikarjeva 101, 1000 Ljubljana, Slovenia

Full list of author information is available at the end of the article
}

(c) The Author(s) 2020. This article is licensed under a Creative Commons Attribution 4.0 International License, which permits use, sharing, adaptation, distribution and reproduction in any medium or format, as long as you give appropriate credit to the original author(s) and the source, provide a link to the Creative Commons licence, and indicate if changes were made. The images or other third party material in this article are included in the article's Creative Commons licence, unless indicated otherwise in a credit line to the material. If material is not included in the article's Creative Commons licence and your intended use is not permitted by statutory regulation or exceeds the permitted use, you will need to obtain permission directly from the copyright holder. To view a copy of this licence, visit http://creativeco mmons.org/licenses/by/4.0/. The Creative Commons Public Domain Dedication waiver (http://creativecommons.org/publicdomain/ zero/1.0/) applies to the data made available in this article, unless otherwise stated in a credit line to the data. 
gene products required for the production of CHD in a genetically amenable heterologous host, thus opening new possibilities towards production of novel and potent tetracycline analogues with a new mode of action.

Keywords: Antibiotics, Polyketide, Heterologous expression, Actinobacteria, Natural product biosynthesis, Tetracyclines, Chelocardin

\section{Background}

Facing increasingly spreading antimicrobial resistance and a severe shortage of novel anti-infectives, one of the strategies to develop new antibiotics is the revival and chemical optimisation of validated chemical scaffolds, such as the tetracyclines (TCs). We previously reported cloning of the biosynthetic gene cluster (BGC) encoding the biosynthesis of the atypical TC, chelocardin (CHD) [1]. CHD belongs biosynthetically to the group of aromatic polyketides which are biosynthesized through decarboxylative condensation of malonate building blocks and subsequent directed cyclization of the nascent precursor polyketide chain thereby resulting in a tetracyclic scaffold [2]. The CHD scaffold itself is further chemically decorated by so called post-PKS enzymes such as oxygenases, methyltransferases and aminotransferases [3], yielding structurally relatively small, but important differences in the structure of CHD compared to the typical TCs. These modifications include a different aromatization pattern of the TC backbone, an acetyl instead of a carboxamido group at carbon 2 [C2], a primary instead of tertiary amine at $\mathrm{C} 4$ in opposite configuration, and an additional methyl group at carbon C9 (Fig. 1). While it is apparent that these differences are reflected in a different mode of action of CHD compared to typical TCs [4, 5], the molecular target of CHD has not been identified yet. We have demonstrated that it is possible to carry out modifications of CHD through genetic engineering of its producer, the actinomycete Amycolatopsis sulphurea. However, A. sulphurea is difficult to genetically manipulate and therefore engineering of the chd biosynthetic gene cluster in a genetically amenable heterologous host would be of high importance for further drug-discovery efforts.

CHD has caught our attention as it exhibits a broadspectrum antibiotic activity and showed promising results in a small phase II clinical study conducted on

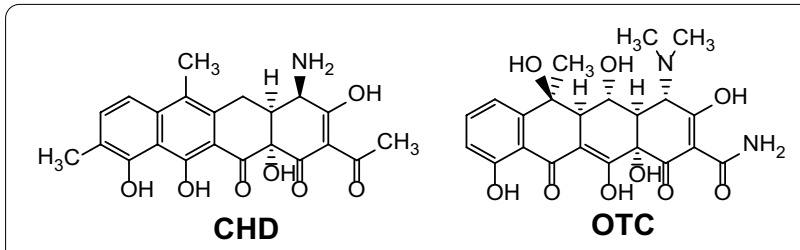

Fig. 1 Structures of CHD and OTC patients with urinary tract infections caused by Gramnegative pathogens in 1977 [6]. CHD was shown to be active also against TC-resistant pathogens, including Gram-negative bacteria [7], which is gaining even more importance in today's antibiotic crisis.

In the recent years, we have demonstrated that modifications of CHD through genetic engineering of its producer, the actinomycete $A$. sulphurea, are not only possible but yielded even more potent antibiotics than CHD itself, like 2-carboxamido-2-deacetyl-chelocardin (CDCHD) [8]. Our work also provided an understanding of the structure-activity relationship of CHD [9]. Furthermore, CDCHD was semi-synthetically modified $[9$, $10]$ with the aim to optimize the potent CHD derivative even further.

Here, we report heterologous expression of the CHD biosynthetic gene cluster in the model organism Streptomyces albus del14 strain [11]. Unexpectedly we here demonstrate that the originally identified CHD BGC [1] fails to provide all genes required for CHD formation, including an additional cyclase and regulatory genes. When comparing to oxytetracycline (OTC) BGC, we have observed that CHD BGC can actually contain genes which are generally not found in typical tetracycline gene cluster. By successful heterologous expression of CHD in a Streptomyces host, we confirmed that the cloned CHD BGC from this work contains all gene products required for regulation, cyclization and formation of the TC backbone of $\mathrm{CHD}$, thus enabling and extending biosynthetic engineering efforts, which were shown previously to be very promising for generating new $\mathrm{CHD}$ analogues, including $\mathrm{CDCHD}$ with potent bioactivity. Considering that gene-manipulation in the native producer $A$. sulphurea is technically demanding, heterologous expression in a genetically amenable actinomycete cell factory would be an important advantage when aiming to generate novel CHD analogues by biosynthetic engineering approaches.

\section{Results and discussion}

\section{Chelocardin biosynthetic gene cluster}

When comparing the published nucleotide sequence of CHD BGC [1], which was based on sequencing of a cosmid selected from a cosmid library, to the A. sulphurea NRRL 2822 whole-genome sequence established in the scope of this work, we found that the original sequence of 
the CHD BGC was misassembled. It turned out that the original cosmid containing the CHD BGC was chimeric and composed of two genomically unlinked fragments (Fig. 2). Correspondingly, we identified an additional DNA fragment containing genes involved in CHD biosynthesis; one additional putative cyclase gene homologue for the biosynthesis of CHD, and two additional putative regulatory genes (Fig. 2). The new biosynthetic gene was designated as $c h d Y$ encoding a putative second ring cyclase protein, a homologue to OxyN from OTC biosynthesis [12]. We also identified a likely mistake in the original CHD BGC sequence, where the C-terminal part of chdOII was not correctly annotated. After correction, the C-terminal part of the chdOII DNA sequence revealed even higher similarity to the oxyL encoding the oxygenase from OTC BGC involved in TC scaffold formation in OTC biosynthesis (Fig. 2) [12]. These corrections were introduced into the current GenBank Accession Number KC870000.

Interestingly, in the CHD BGC, ChdOII and ChdY (Table 1) are encoded as one single polypeptide, thus forming a bifunctional protein. In contrast, two separate ORFs encoding OxyL and OxyN (homologues of ChdOII and ChdY, respectively) are present in OTC BGC. Analogously, this is also observed for chdL and chdOIII (Table 1) nucleotide sequences from CHD BGC, which also yield one single polypeptide. Similarly, chdL and chdOIII homologues from OTC BGC, oxyH and oxyG, respectively, are translationally coupled (Table 1).

Finally, bioinformatic analysis of the DNA sequence downstream of the newly-identified putative cyclase gene $c h d Y$ revealed two regulatory genes, $c h d B$ and chdC, encoding SARP and LuxR type regulators, respectively (Table 1). chdB and chdC homologues, otcR and otcG, respectively, are also identified in OTC and chlortetracycline BGCs in S. rimosus and S. aureofaciens, respectively (Fig. 2) [13, 14].

\section{Proposed CHD biosynthetic pathway}

Considering the additional part of CHD BGC, we repeated the analysis of the $\mathrm{CHD} \mathrm{BGC}$ and re-assigned the putative biosynthetic steps (Fig. 3) in comparison to the biosynthetic pathway of OTC $[2,12]$. We focused our study on the putative gene homologues involved in formation of the basic TC scaffold. Thus, when comparing early stages in OTC and CHD biosynthesis, gene homologues can be identified in both BGCs, which are necessary for the formation of early putative biosynthetic intermediates such as 4-keto-ATC in OTC pathway (8, Fig. 3), strongly resembling the putative CHD precursor, 4-keto-9-desmethyl-CHD (8, Fig. 3). These two intermediates differ only in the moiety attached at the $\mathrm{C} 2$ position, as a consequence of incorporation of a different starter unit. However, it is important to mention a known congener in OTC biosynthesis (8, Fig. 3) leading to a well-known impurity, 2-acetyl-2-decarboxamido-OTC (AD-OTC, [12]), which is presumably primed by the same starter unit as in CHD biosynthesis. Analogously, in $\mathrm{CHD}$ biosynthesis, $\mathrm{AD}-\mathrm{OTC}$ should then be structurally identical to 4-keto-9-desmethyl-CHD (8, Fig. 3).

The enzymes putatively involved in CHD biosynthesis are marked in blue colour. Similarly, the possible intermediate structures, belonging exclusively to the CHD biosynthetic pathway branch are labelled in blue colour. Finally, numbering of carbon atoms is according to chemical nomenclature.

The formation of the TC backbone in CHD is catalyzed by the type II minimal polyketide synthase (minimal PKS) genes, consisting of ketosynthase $\alpha$, ketosynthase $\beta$ and acyl carrier protein (ACP), designated as ChdP, ChdK and

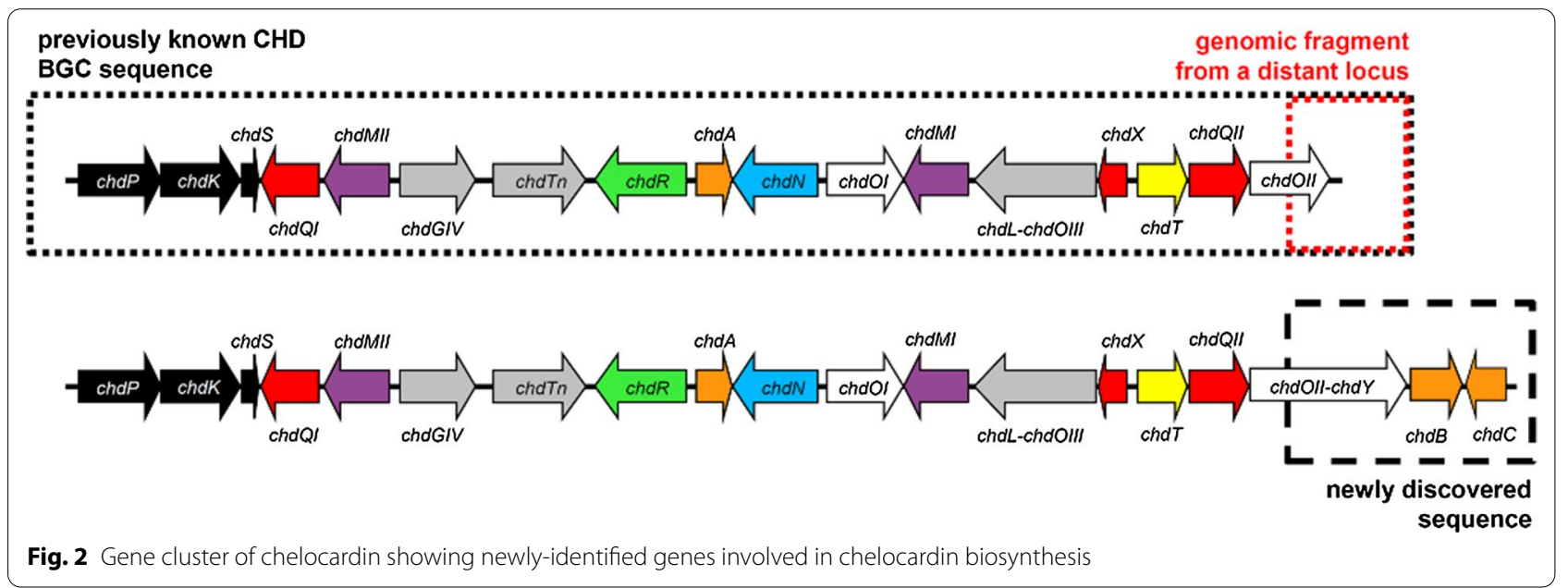


Table 1 Proposed function of gene homologues identified in the CHD BGC

\begin{tabular}{|c|c|c|c|c|}
\hline Gene & Size (AA) & Putative function & $\begin{array}{l}\text { Protein homologue (GenBank } \\
\text { Accession Number) }\end{array}$ & $\begin{array}{l}\text { Identity/ } \\
\text { similarity } \\
(\%)\end{array}$ \\
\hline chdP & 432 & Ketosynthase alpha & OxyA (P43678.2) & $78 / 85$ \\
\hline chdK & 418 & Ketosynthase beta & OxyB (AAZ78326.1) & $71 / 82$ \\
\hline chds & 88 & Acyl carrier protein & OxyC (P43677.1) & $62 / 77$ \\
\hline chdQI & 302 & Aromatase/cyclase & OxyK (AAZ78334.2) & $32 / 48$ \\
\hline chdMII & 343 & Methyltransferase & CmmMII (CAE17532.1) & $45 / 61$ \\
\hline chdGIV & 399 & Glycosyltransferase & CmmGIV (CAE17547.1) & $39 / 51$ \\
\hline chdTn & 511 & Transposase & InsG (P03835.1) & $25 / 40$ \\
\hline $\operatorname{chdR}$ & 481 & Exporter & EmrB (P9WG88.1) & $33 / 54$ \\
\hline $\operatorname{chdA}$ & 190 & Transcriptional regulator & TetR (5MRU_A) & $43 / 56$ \\
\hline$c h d N$ & 448 & Aminotransferase & Msat (1WST_A) & $31 / 47$ \\
\hline chdOl & 404 & Oxygenase & OxyE (AAZ78329.1) & $66 / 78$ \\
\hline chdMl & 341 & Methyltransferase & OxyF (AAZ78330.1) & $66 / 76$ \\
\hline chdL-chdOIII & 643 & Acyl-CoA ligase/oxygenase & OxyH (ELQ83297.1) & $56 / 69$ \\
\hline chdOIII & 96 & Oxygenase & OxyG (AAZ78331.1) & $63 / 76$ \\
\hline $\operatorname{chd} X$ & 150 & Cyclase & Oxyl (AAZ78332.2) & $64 / 74$ \\
\hline chdT & 262 & Ketoreductase & OxyJ (AAZ78333.1) & $76 / 85$ \\
\hline chdQII & 315 & Aromatase/cyclase & OxyK (AAZ78334.2) & $58 / 67$ \\
\hline chdOIl-chdY & 806 & Oxygenase/cyclase & OxyL (AAZ78335.1) & $59 / 69$ \\
\hline$c h d Y$ & 256 & Cyclase & OxyN (AAZ78337.1) & $73 / 80$ \\
\hline $\operatorname{chdB}$ & 258 & Transcriptional activator (SARP family) & OtcR (AJO26937.1) & $45 / 63$ \\
\hline chdC & 212 & Transcriptional activator (LuxR family) & OtcG (ACM67367.1) & $35 / 56$ \\
\hline
\end{tabular}

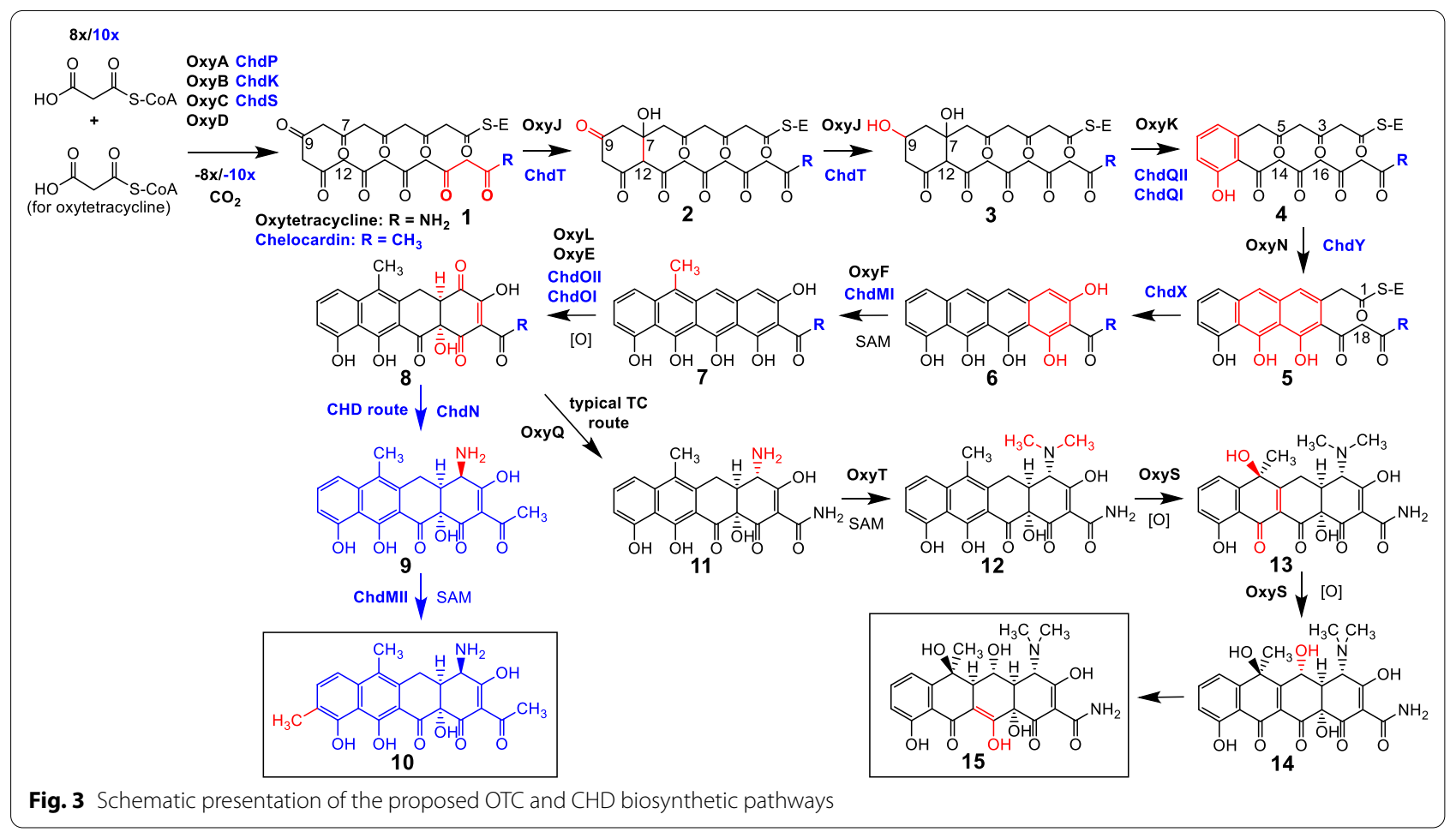


ChdS, respectively. It is expected that the minimal PKS of CHD condenses 10 malonyl-CoA building blocks into an acetate-primed decaketide (1, Fig. 3) [1]. The malonyl$\mathrm{CoA} A \mathrm{ACP}$ acyltransferase required for the transfer of the extender unit malonyl-CoA to the ACP, was proposed to be shared with fatty acid biosynthesis [15]. Analogously as in OTC biosynthesis, catalyzed by OxyJ [12], initial folding of the growing polyketide chain in CHD is most probably directed by the ketoreductase ChdT, resulting in reduction of the keto group at C9 (3, Fig. 3). Closure of 4 rings leading to the formation of the CHD backbone is most likely directed by aromatases/cyclases ChdQI, ChdQII, ChdY and ChdX. The first two are both similar to OxyK, and the latter two are homologous of OxyN and OxyI, respectively [2,12]. Based on homologies to aromatases/cyclases encoded in other aromatic polyketide BGCs, we propose that the didomain aromatase/ cyclases ChdQI and ChdQII are both responsible for formation of first ring D (4, Fig. 3), while the monodomain cyclase ChdY is needed for the second ring $(\mathrm{C})$ closure. As is the case in biosynthesis of other aromatic polyketides, formation of third ring (B) could be spontaneous (5, Fig. 3) or potentially catalyzed by second ring cyclase as suggested by Hertweck et al. $[3,16]$. Possibly, the last ring (A) cyclization is carried out by the cyclase ChdX (6, Fig. 3). We have proposed the function of the putative cyclase ChdX based on the comparison with chromomycin and mithramycin biosynthetic pathways [17]. The function of its homologue in OTC biosynthesis, OxyI, on the other hand, has not yet been elucidated and was shown that it is not essential for last ring closure due to the terminal amide moiety, potentially leading to spontaneous cyclization $[12,16]$. Interestingly, it was proposed by Pickens et al. that acyl-CoA-ligase SsfL2, OxyH homologue from the OTC BGC, is involved in the formation of the fourth ring of the TC-like compound SF2575 [12, 18]. Indeed, it was later demonstrated that SsfL2 catalyzes an ATP-dependent $\mathrm{C} 1-\mathrm{C} 18$ Claisen condensation to close the fourth ring in the biosynthesis of SF2575 [19, 20]. The nascent TC scaffold undergoes further processing towards the final structure of CHD by so-called postPKS tailoring reactions. Compared to OTC biosynthesis, the late steps in CHD biosynthesis, namely putative C-9 methylation by ChdMII and transamination of $\mathrm{C} 4$ in opposite stereochemistry catalyzed by $\mathrm{ChdN}$, respectively, are the last two steps in CHD biosynthesis and significantly differ from the biosynthesis of typical TCs such as OTC (Fig. 3). The putative C6-methyltransferase ChdMI is a homologue of OxyF from OTC BGC [16], (7, Fig. 3). Putative oxygenases ChdOI and ChdOII, homologues of OxyE and OxyL from OTC BGC [21], respectively, carry out hydroxylation of ring $\mathrm{A}$ at $\mathrm{C} 4$ and $\mathrm{C} 4 /$ $\mathrm{C} 12 \mathrm{a}$, respectively (8, Fig. 3). Oxidation at $\mathrm{C} 4$ results in formation of a chinone which is reductively transamidated by ChdN (9, Fig. 3), a PLP-dependent aminotransferase. Surprisingly, although catalyzing almost identical reactions, ChdN is only distantly related to OxyQ, which is responsible for incorporation of the amino group at $\mathrm{C} 4$ in OTC biosynthesis; however, the biosynthesis results in opposite stereochemistry at C4 [12]. The activity of these two divergent aminotransferases represents a branching point between CHD and typical TCs biosynthesis and results in different products. The amino group incorporated into CHD is installed in $R$-configuration, while the one in OTC biosynthesis is in $S$-configuration. In contrast to the more decorated backbone of typical TCs such as OTC, there is only one more tailoring reaction leading to CHD: C9-methylation. This reaction is believed to be catalyzed by ChdMII (10, Fig. 3), a homologue of the C9-methyltransferases from chromomycin and mithramycin biosynthesis [17].

Overall, when comparing the biosynthetic pathways of $\mathrm{CHD}$ and OTC, a very high degree of similarity between the genes can be observed (Fig. 3). However, a number of genes present in the CHD BGC do not have their counterparts in typical TC BGCs, which particularly relates to the C-9 methyltransferase chdMII, the C4-aminotransferase $c h d N$ and the exporter/resistance gene $c h d R$, thus suggesting that the CHD gene cluster evolved by incorporating genes from aureolic acids type II PKS systems such as chromomycin and mithramycin.

When comparing enzymes involved in the early steps of biosynthesis of the basic TC backbone, with exception of starter unit selection and cyclisation of the ring $\mathrm{C}$. The cyclisation of the C-ring in CHD biosynthesis is somehow different, considering that a fully aromatic structure is maintained in CHD biosynthesis (Fig. 1). In OTC biosynthesis, where only D ring is aromatized (Fig. 1), hydroxylation of the positions $\mathrm{C} 5$ and $\mathrm{C} 6$ is carried out by OxyS and OxyR [22]. CHD BGC does not contain OxyS and OxyR homologues, thus no further hydroxylation occurs in CHD biosynthesis at $\mathrm{C} 5$ and $\mathrm{C} 6$ positions, and this is probably also a reason that CHD maintains ring $\mathrm{C}$ aromatized $[1,23]$.

The aromatization pattern of $\mathrm{C}$ and $\mathrm{D}$ rings likely has direct influence on the overall planarity of the backbones of CHD and OTC [24], with implications regarding the different mode of action of CHD. As discussed earlier, an important branching point in the biosynthetic pathways of OTC and CHD is most likely the addition of the amino group with opposite stereochemistry, carried on the putative quinoide intermediate harboring a keto-group at carbon C4 (8, Fig. 3). This step also has a very important implication for the activity of CHD [8], in addition to carbon $\mathrm{C} 9$ methylation of the CHD backbone, which does not occur in the OTC biosynthesis $[8,12]$. 
Regulation of CHD biosynthesis and self-resistance

One of the putative regulatory proteins found in CHD BGC (Fig. 2), ChdB, is a close homologue to OtcR in OTC biosynthesis [14]. OtcR is a positive regulatory protein, close homolog of Streptomyces antibiotic regulatory protein (SARP) from oxytetracycline, SF2575, dactylocycline and chlortetracycline BGC, OtcR, SsfT1, DacT1, $\mathrm{CtcB}$, respectively as presented on the Additional file 1: Figure S1. OtcR is acting as a pathway-specific activator in OTC biosynthesis in $S$. rimosus and leading to a significant increase in OTC production when overexpressed at the appropriate level [14].

The second putative regulatory protein ChdC, found in the CHD BGC belongs to the LuxR family of regulatory proteins and shows high homology to regulatory protein OtcG from OTC biosynthesis, identified by Lešnik et al. [13]. Close homologs of ChdC are found in oxytetracycline, dactylocycline and chlortetracycline BGC, OtcG, DacT3 and CtcA, respectively are presented on Additional file 1: Figure S2. In OTC biosynthesis, OtcG has a conditionally positive regulatory role in OTC production. As demonstrated by Lesnik et al., otcG inactivation reduced the production of OTC by more than $40 \%$, while its overexpression under a strong constitutive promoter did not yield any statistically significant change in the production of OTC [13]. Therefore, in the scope of this study, we mostly focused on the SARP transcription activator $\mathrm{ChdB}$,

Finally, chdR encodes a putative integral membrane protein that is most probably responsible for the efflux of $\mathrm{CHD}$ from the cell and is probably regulated by another regulatory protein, the putative TetR family repressor protein ChdA [1]. Interestingly, despite the relatively high structural similarity between OTC and CHD structures, ChdR from CHD BGC does not share high homology with the OtrB exporter from OTC BGC, suggesting significant differences in mode of action and the structure of TCs and CHD. The resistance to CHD may have a significant impact on the production of antibiotic in heterologous host. Therefore, we intended to carry out in-trans over-expression of $c h d R$ gene, which is likely providing resistance to $\mathrm{CHD}$ and $\mathrm{CD}-\mathrm{CHD}$ in $A$. sulphurea and thus, may also have an important role in the heterologous host.

\section{Overexpression of streptomyces antibiotic regulatory protein (SARP) chdB gene}

We carried out the overexpression of an additional copy of SARPs $c h d B$ and $o t c R$ from S. rimosus [14] in native producer $A$. sulphurea as described in materials and methods. The selected genes were placed under expression of ActIIORF4/PactI activator/promotor system [25], resulting in pAB03otcR and pAB03-SARP (Additional file 1: Table S2). A ФBT- based integrative vector pAB03 was used for in-trans integration of the selected genes.

Overexpression of native SARP $c h d B$ clearly increased the yield of CHD and CDCHD for 1.5- to 1.9-fold, respectively (Additional file 1: Table S3). However, additional copy of SARP homologue of otcR from S. rimosus M4018, did not have significant positive effect on CHD production. In contrast, when $o t c R$ was overexpressed in the CDCHD-producing strain of $A$. sulphurea, there was clearly a positive effect on the production of target compound (Fig. 4). Thus, we can confirm that $c h d B$ is a positive regulatory protein in $\mathrm{CHD}$ biosynthesis.

\section{Cloning and heterologous expression of CHD BGC using replicative and integrative cosmids}

After screening of the newly constructed A. sulphurea cosmid library based on the replicative conjugative cosmid vector pOJ456, a cosmid containing the entire CHD BGC was identified and designated as pOJ456-CHD12. Cosmid library construction and the subsequent cloning and heterologous expression procedures are described in the materials and methods section. The selected cosmid pOJ456-CHD12 was introduced into S. albus del14 [11] by conjugation in an attempt to heterologously express the CHD BGC. The model organism S. albus was selected as a heterologous host considering, this strain is significantly more amenable to genetic manipulations than the native producer $A$. sulphurea, which would help in faster development of new analogues. $S$. albus del14 strain, developed by Myronovskyi et al., was optimized even further by deletion of its native clusters encoding secondary metabolite biosynthetic pathways [11].

However, by applying liquid chromatography-mass spectroscopy (LC-MS) analysis no CHD could be detected in culture broth extracts of $S$. albus del14 carrying cosmid pOJ456-CHD12, which could be due to instability of the replicative cosmid or CHD self-resistance issues. Therefore, we transferred the entire CHD BGC into an integrative cosmid pOJ436, containing the ФC31 integrase, to allow a stable integration of CHD BGC into the genome of $S$. albus del14. Indeed, heterologous expression of CHD BGC from chromosomally integrated cosmid pOJ436-CHD12 was successful and resulted in the production of $\mathrm{CHD}$, reaching a yield of around $50 \mathrm{mg} / \mathrm{L}$ (Fig. 5, Table 2).

\section{Attempt to increase the production yield of CHD in a heterologous host through overexpression of chdR efflux pump gene}

Considering unusual mode of action of CHD [26], which is still not clearly understood, and in an attempt to increase yield of CHD by overcoming possible self-resistance issues during heterologous expression of CHD, we 


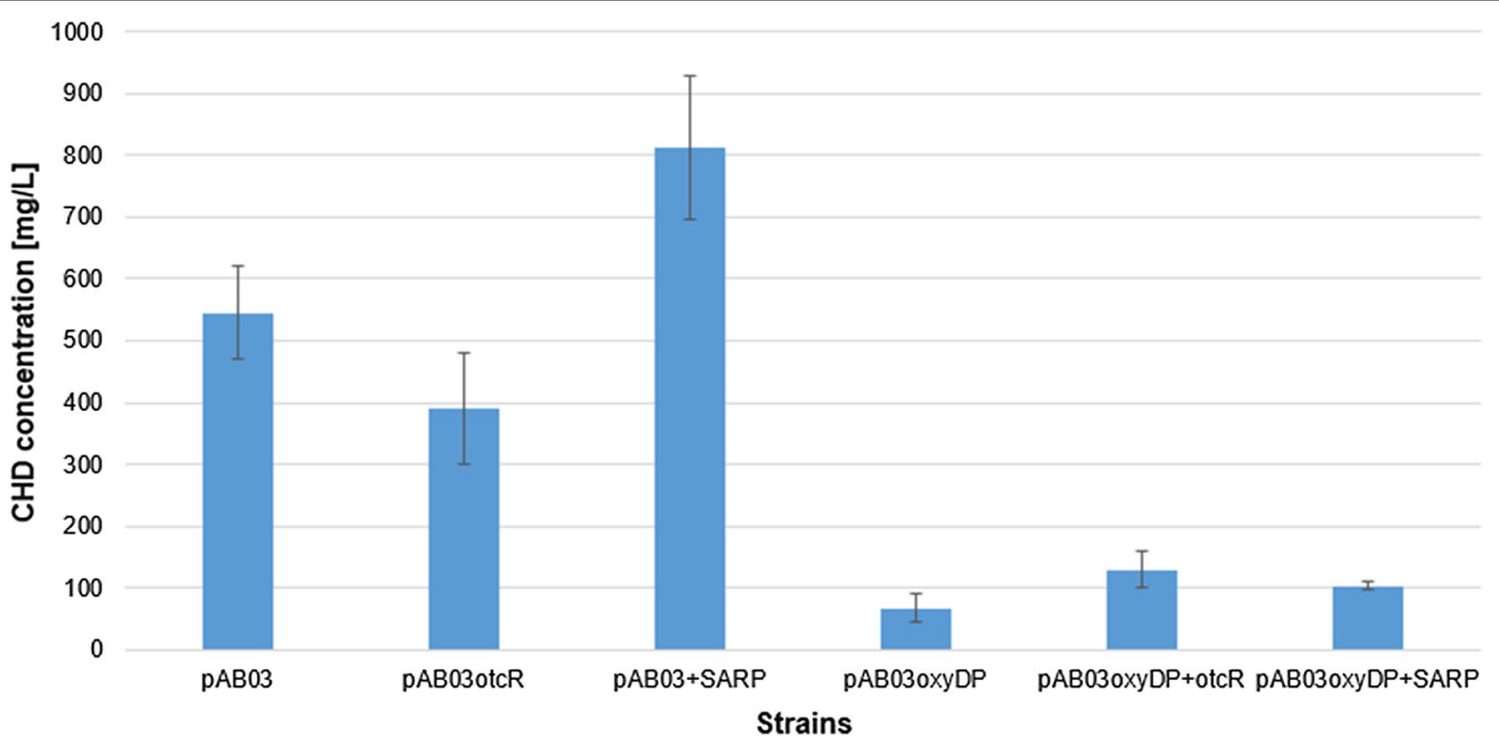

Fig. 4 Overexpression of SARP homologues otcR from S. rimosus and chdB in native producer A. sulphurea

constructed a second integrative cosmid carrying entire CHD BGC with an additional copy of CHD efflux pump gene $c h d R$ under control of the strong constitutive promoter $\mathrm{P}_{\text {ermE* }}$ (construct pOJ436-PermE*-chdR-CHD12). However, the additional copy of efflux pump gene $c h d R$ did not lead to a significant increase of production yields of CHD in S. albus del14, reaching up to $60 \mathrm{mg} / \mathrm{L}$ (Fig. 5, Table 2).

\section{Production of CDCHD in the heterologous host}

We also aimed to produce CDCHD [8] as this compound exhibits superior antibacterial activity when compared to CHD, including activity against all Gram-negative pathogens of the ESKAPE panel [8]. To achieve this goal, we constructed two integrative cosmids; one carrying CHD $\mathrm{BGC}$ and oxyDP (genes for amidotransferase OxyD and acyltransferase OxyP from OTC BGC) and the second plasmid $o x y D P c h d R$ carrying genes for amidotransferase OxyD and acyltransferase OxyP from OTC BGC and an additional copy of efflux pump ChdR from CHD BGC, which were expressed under strong promoter $\mathrm{P}_{\text {ermE* }}$ (constructs pOJ436-PermE*-oxyDP-CHD12 and pOJ436PermE*-oxyDPchdR-CHD12, respectively). Although at relatively low yield of approximately $3 \mathrm{mg} / \mathrm{L}$, production of CDCHD was achieved in some $S$. albus del14 ex-conjugants with integrated pOJ436-PermE*-oxyDPchdR-CHD12 (Fig. 6, Table 2). Interestingly, production of CDCHD was not achieved in S. albus del14 ex-conjugants with integrated cosmid without the additional copy of efflux pump ChdR (construct pOJ436-PermE*-oxyDPCHD12), suggesting lack of self-resistance by the engineered S. albus strains towards CDCHD.

\section{Conclusion}

Although structurally similar to typical TCs such as OTC, CHD displays a different mode of action as antibacterial compound [27]. We have previously reported cloning of the respective BGC [1]. However, the entire genome sequence of producer strain $A$. sulphurea, which we have determined here, revealed an additional fragment of the CHD BGC containing a putative second ring cyclase gene homologue, which allowed us to explain previously puzzling biosynthetic steps of the early steps in CHD biosynthesis. Additionally, two more putative regulatory genes were identified, opening potential to aid in efforts to increase the production yield of CHD and other potential analogues of CHD such as CDCHD. In light of these new findings, we attempted to produce CHD in a genetically amenable heterologous host S. albus. The cloning of the entire CHD BGC and expression in S. albus on a single cosmid was successful. In addition, biosynthetic genes oxyD and oxyP from OTC BGC, involved in the supply of malonamate starter unit, were added to the cosmid carrying entire CHD BGC, to ensure production of the more potent $\mathrm{CHD}$ analogue, $\mathrm{CDCHD}$, which is currently showing promising activity in preclinical evaluation [10].

When considering structure-activity correlation, the basic TC backbone of CHD contains two aromatized rings (D and $\mathrm{C}$ ) in contrast to the OTC structure, which only has the first ring (D) aromatized. This seemingly small difference in the structure of the basic TC backbone however, most likely has a profound influence on the planarity, where CHD with two rings aromatized bears a more planar structure. It is also important to consider that there are significant differences in CHD 


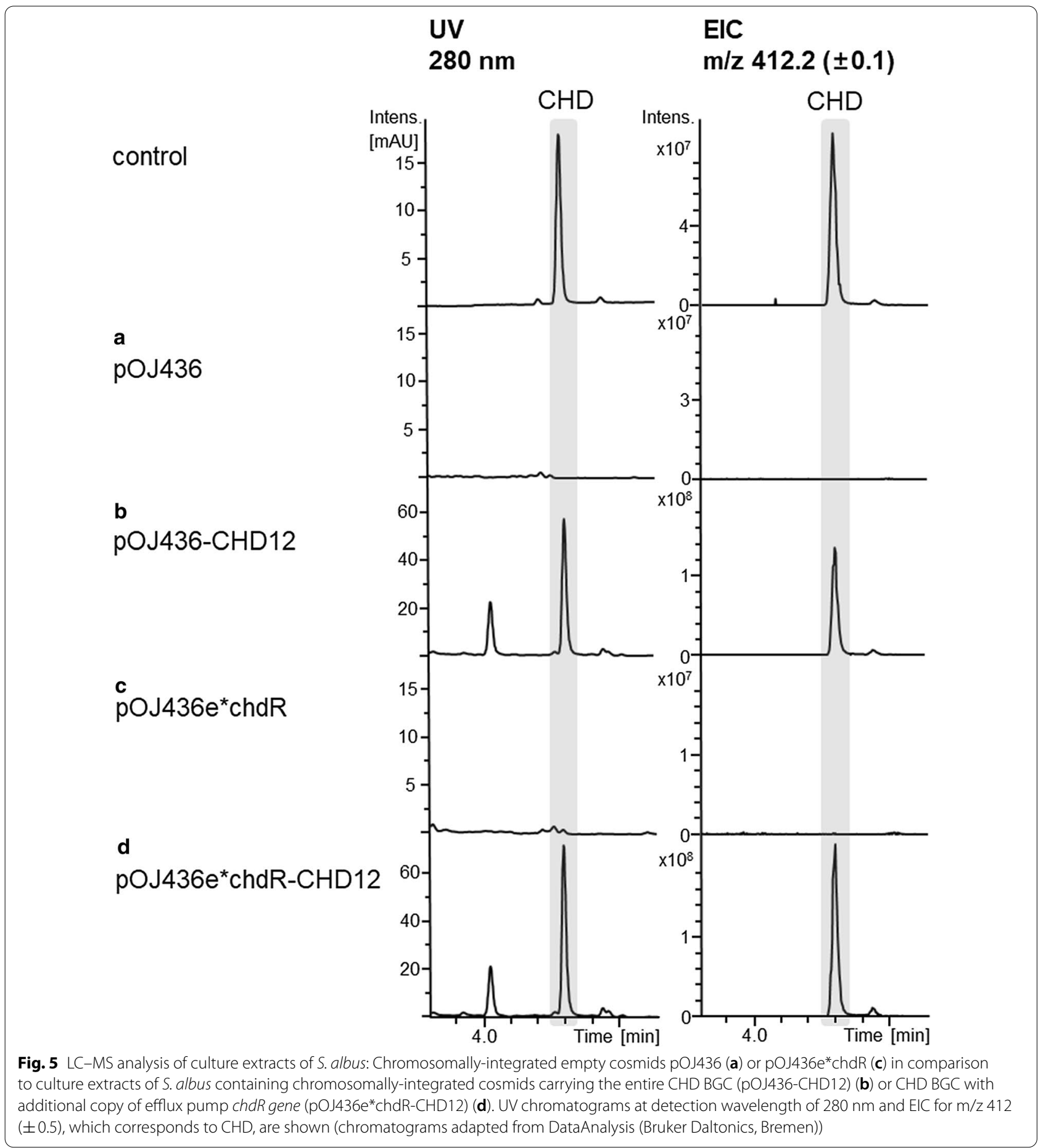

and OTC BGCs, which lead to different moieties being incorporated into the basic TC scaffold. These include enzymes catalyzing priming steps, and late biosynthetic enzymes involved in methylation, addition of amino and hydroxyl groups at $\mathrm{C} 9, \mathrm{C} 4, \mathrm{C} 6$ and $\mathrm{C} 5$, respectively. C4 transamination, which occurs in opposite stereochemistry in both compound classes, can be considered as a branching point between CHD and TC biosynthesis (Fig. 3). At this point, CHD and OTC pathways split towards typical and atypical TCs, such as OTC and CHD biosynthesis, respectively (Fig. 3). Absence of C6 hydroxylation, di-methylation of the $\mathrm{C} 4$ amino group and 
Table 2 Description of the S. albus del14 engineered strains for heterologous expression of CHD BGC, and corresponding product yields

\begin{tabular}{|c|c|c|c|c|c|c|}
\hline Introduced cosmid & ChdR & OxyD & OxyP & CHD BGC & Product & Yield \\
\hline pOJ456-CHD12 & & & & $\checkmark$ & / & / \\
\hline pOJ436-CHD12 & & & & $\checkmark$ & $\mathrm{NH}_{2}$ & Up to $50 \mathrm{mg} / \mathrm{L}$ \\
\hline pOJ436-PermE*-chdR-CHD12 & $d$ & & & $\checkmark$ & & Up to $60 \mathrm{mg} / \mathrm{L}$ \\
\hline pOJ436-PermE*-oxyDP-CHD12 & & $\checkmark$ & $\checkmark$ & $\checkmark$ & / & / \\
\hline pOJ436-PermE*-oxyDPchdR-CHD12 & $\checkmark$ & $\checkmark$ & $\checkmark$ & $\checkmark$ & & $\sim 3 \mathrm{mg} / \mathrm{L}$ \\
\hline
\end{tabular}

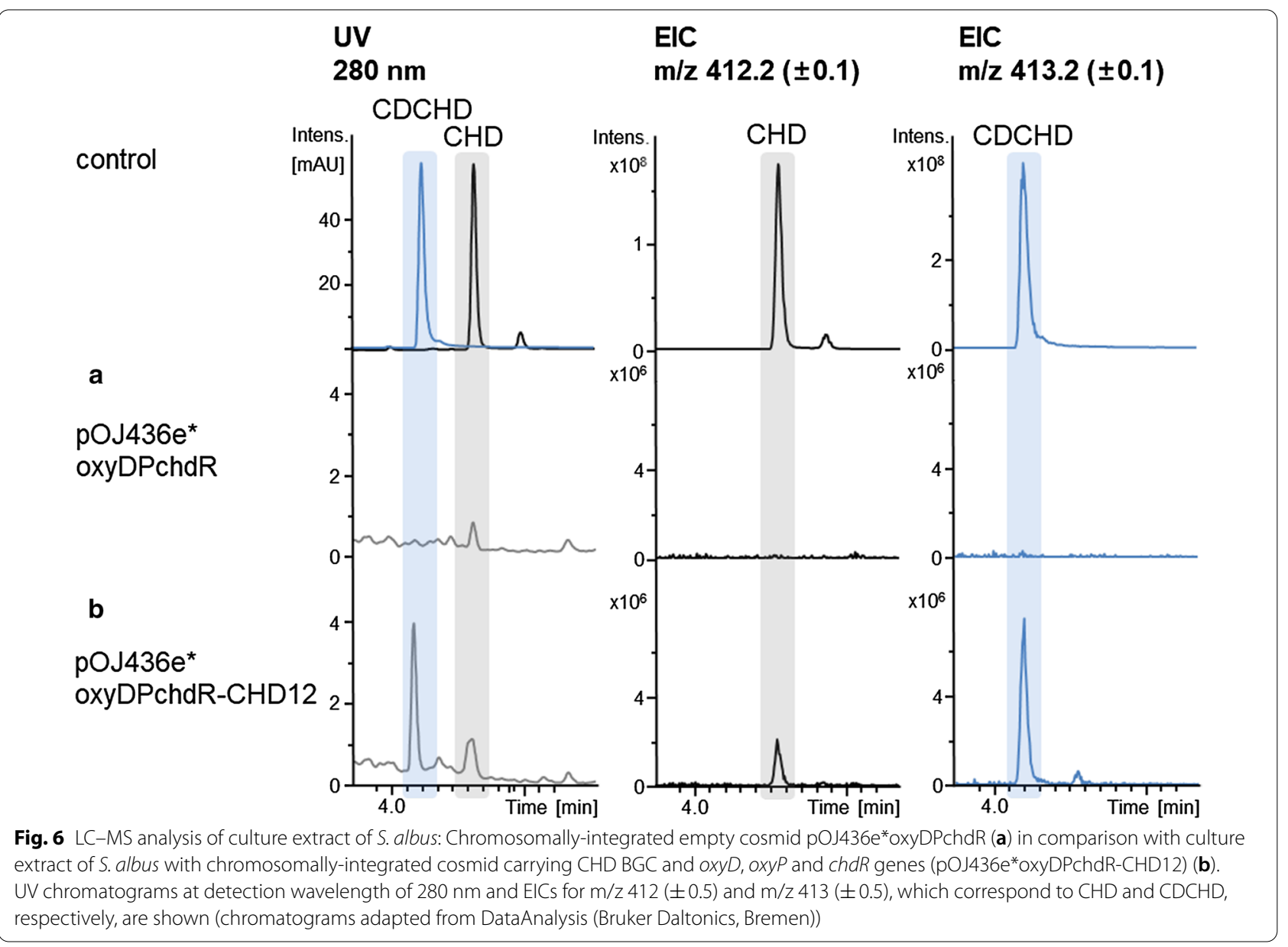


presence of additional C9 methylation in CHD biosynthesis may also result in a more planar structure of CHD, hence contributing to its potent activity and bactericidal mode of action (in contrast to bacteriostatic TCs acting on ribosomes).

Studies comparing the BGCs of CHD and OTC also revealed some evolutionary aspects of the development of type II PKS enzyme complexes (Fig. 2). Interestingly, when analyzing the entire CHD BGC from A. sulphurea, and comparing it to the other type II PKS, we observed that some areas in the CHD BGC encoding the C-9 methyltransferase chdMII and the glycosyl-transferase chdGIV, (Fig. 2) do show high homology to aureolic acids BGCs, such as those encoding mithramycin and chromomycin. In addition, we have also identified four genes, including the $c h d N$ carbon- 4 aminotransferase and the exporter $\operatorname{chdR}$ (Fig. 2), which do not show any significant homology to other genes found in type-II PKS BGC. Although CHD seems structurally very similar to typical TCs, these genes make CHD distinct in structural properties and particularly in biological activity.

In this study we identified additional essential genes for CHD biosynthesis, which was confirmed by their heterologous expression in S. albus as heterologous host. The corrected nucleotide sequence of the CHD BGC therefore contains all gene products required for the production of CHD. The heterologous system is significantly more amenable to genetic manipulation, thus opening new possibilities towards production of novel and potent TC analogues exhibiting a new mode of action.

\section{Methods}

\section{Bacterial strains and culture conditions}

Amycolatopsis sulphurea NRRL 2822 (ARS Culture Collection) was used for production of chelocardin (CHD) and as a source of DNA and for microbiological manipulations. Streptomyces rimosus M4018 [28] was used as a source of DNA for cloning of genes from oxytetracycline (OTC) biosynthetic gene cluster (BGC). Streptomyces albus del14 [11] was used for heterologous expression of CHD BGC. Escherichia coli DH10 $\beta$ (Invitrogen) was used for standard cloning procedures [29], and E. coli GB2006 (Gene Bridges) for preparation of A. sulphurea cosmid library. E. coli ET12567 [30] carrying pUZ8002 plasmid [31] was used as a donor strain for intergeneric conjugation with S. albus del14 (Additional file 1: Table S1). Soya mannitol (MS) agar and tryptone soy broth (TSB) [32] were used as agar sporulation medium and for cultivation of actinomycetes in liquid medium, respectively. For heterologous production of CHD and CHD analogues, $S$. albus was cultivated in TSB seed medium and four different production media: CH-F2, DNPM (4\% dextrin, $0.75 \%$ soytone, $0.5 \%$ baking yeasts, $2.1 \%$ MOPS, pH 6.8 [33]),
NL5Y $\left(0.1 \% \mathrm{NaCl}, 0.1 \% \mathrm{KH}_{2} \mathrm{PO}_{4}, 0.05 \% \mathrm{MgSO}_{4} \times 7 \mathrm{H}_{2} \mathrm{O}\right.$, $2.5 \%$ glycerol, $0.584 \%$ L-glutamine, $0.2 \%$ trace elements solution, $1 \%$ yeast extract, pH 7.3 [34], and SG1 (2\% glucose, $0.5 \%$ yeast extract, $1 \%$ soytone, $0.2 \% \mathrm{CaCO}_{3}, \mathrm{pH}$ 7.2 [35]). Cultivations were performed in Falcon tubes at $30{ }^{\circ} \mathrm{C}$ on a rotary shaker at $220 \mathrm{rpm}$ for $36 \mathrm{~h}$ in seed medium with $5 \%(\mathrm{v} / \mathrm{v})$ used to inoculate production media and cultivated for further 7 days under the same conditions. For intergeneric conjugation between $S$. albus and E. coli MS medium, supplemented with $10 \mathrm{mM}$ $\mathrm{MgCl}_{2}$ was used. Apramycin $(50 \mu \mathrm{g} / \mathrm{mL})$ together with nalidixic acid $(25 \mu \mathrm{g} / \mathrm{mL})$ was used for selection of $S$. albus exconjugants on MS. For selection of E. coli transformants, Apr $(50 \mu \mathrm{g} / \mathrm{mL})$, Kan $(25 \mu \mathrm{g} / \mathrm{mL})$ or chloramphenicol $(\mathrm{Cm} ; 10 \mu \mathrm{g} / \mathrm{mL})$ were added into LB medium.

\section{Overexpression of SARPs in A. sulphurea}

Genes otcR from S. rimosus M4018 and $c h d B$ from A. sulphurea were PCR amplified (Additional file 1: Table S2) using otcR-Fw, OtcR-Rv and SARP-Fw, SARPRv primers, respectively. The PCR amplified DNA fragments were digested with $\mathrm{NdeI}$ and $\mathrm{XbaI}$ and separately cloned into pAB03, downstream of the actI promoter under control of the ActIIORF4/PactI activator/promotor system [25], resulting in pAB03otcR and pAB03SARP (Additional file 1: Table S2), respectively. When the DNA fragments were intended for cloning into pABoxyDP plasmid, they were digested only with $\mathrm{Xba \textrm {I }}$, which retained additional RBS sequence in front of the gene, yielding pAB03oxyDPotcR and pAB03oxyDP-SARP (Additional file 1: Table S2). Plasmids were used to transform E. coli SCS110 and together with pAB03 introduced into $A$. sulphurea via direct transformation of mycelium [36]. A ФВТ- based integrative pAB03 vector stably integrates into the chromosome of A: sulphurea. S27M plates were overlaid with Apr after 16-h incubation. Each transformant colony was further repatched onto MS agar containing Apr and incubated for 10 days. Transformants were inoculated in CHD-V medium followed by CHD-F2 medium, as described above. CHD and CDCHD were extracted from production broth and production yields were measured using LC-MS.

\section{DNA isolation and manipulation}

Isolation and manipulation of DNA in E. coli were carried out according to standard protocols. [29, 32] Cosmids were introduced into $S$. albus del14 via conjugation [32]

\section{Sequencing of genomic DNA}

Salting out procedure [32] was used to isolate genomic DNA from A. sulphurea which was submitted for sequencing with Illumina technology to Seq-IT 
(Kaiserslautern, Germany). Two separate sequencing libraries were prepared (paired-end, mate-pair) which were used for cluster generation on a single lane of MiSeq instrument (50\%/50\% fill rate). Obtained raw sequencing read data was then assembled with the help of abyss-pe 1.3.6 software [37]. The estimated genome size of $A$. sulphurea NRRL 2822 is $7.0 \mathrm{mbp}$ and was contained in 126 contigs on 8 scaffolds.

\section{Preparation of A. sulphurea cosmid library}

Genomic DNA was partially digested with Sau3AI and the DNA fragments of approximate size $35-40 \mathrm{~kb}$ were ligated into the $B a m H \mathrm{I}$ site of replicative conjugative cosmid vector pOJ456, a modified version of the pOJ436 vector [38], where $2.5 \mathrm{~kb}$ ФC31 integrase cassette was excised with HindIII (overhangs were filled in with Klenow polymerase) and replaced with $2.5 \mathrm{~kb}$ pSG5 replication cassette excised with Eco81I and SphI (overhangs were filled in with Klenow polymerase) from medium copy number vector pKC1139 [38]. The ligated DNA was packaged into phage particles (Gigapack III Gold Packaging kit, Agilent Technologies) and introduced into E. coli GB2006.

\section{Identification and sequencing of cosmid carrying CHD BGC} The cosmid library was screened by combining all 3400 colonies and streaking the mixture onto LB agar plates supplemented with $3 \mu \mathrm{g} / \mathrm{mL}$ of $\mathrm{CHD}$ to select for CHD-resistant single colonies expressing ChdR efflux pump encoded in the CHD BGC. 18 positive clones were selected to isolate cosmid DNA and additional PCR screening was carried out using the primer pairs CobU1/CobU2 and glu1/glu2 (Additional file 1: Table S2), designed to anneal to the flanking regions of CHD BGC. Based on the PCR screen, two cosmids were selected for complete sequencing by Illumina sequencing, resulting in confirmation of cosmid pOJ456CHD12, carrying the complete CHD BGC, whose correct and complete sequence was also identified from genomic DNA sequence of A. sulphurea NRRL 2822. The GenBank Accession Number for the revised CHD BGC is KC870000.

\section{Construction of different versions of cosmids carrying CHD BGC}

$34 \mathrm{~kb}$ DNA fragment encoding CHD BGC from pOJ456CHD12 was cloned via SpeI and XbaI into integrative conjugative cosmids pOJ436, pOJ436 $\mathrm{e}^{*} \mathrm{chdR}$, pOJ436e*oxyDP, or pOJ436e $\mathrm{e}^{*}$ oxyDPchdR, resulting in pOJ436CHD12, pOJ436e $\mathrm{e}^{*} \mathrm{chdRCHD12,}$ pOJ436e*oxyDPCHD12, and pOJ436e *oxyDPchdRCHD12, respectively. pOJ436 $\mathrm{e}^{*}$ chdR, $\mathrm{pOJ} 436 \mathrm{e}^{*}$ oxyDP, or pOJ436e oxyDPchdR were constructed from pOJ436 by introducing $1.8 \mathrm{~kb}, 3.2 \mathrm{~kb}$ and $4.7 \mathrm{~kb}$ fragments, carrying chdR, oxyDP and oxyDPchdR genes, respectively, all under the control of $\mathrm{P}_{\text {ermE* }}$ promoter. Fragments were excised with Ecl136II (overhang was filled in with Klenow polymerase) and $X b a \mathrm{I}$ from plasmids pAB03e*chdR, pAB03e*oxyDP and pAB03e*oxyDPchdR, respectively, and used to replace the $1.9 \mathrm{~kb}$ fragment in pOJ436, excised with NruI (overhang was filled in with Klenow polymerase) and $\mathrm{XbaI}$. pAB03e"chdR was constructed by cloning $1.5 \mathrm{~kb}$ chdR gene, amplified by PCR using primers chdRF and chdRR (Additional file 1: Table S2), digested with NdeI and XbaI and ligated into pAB03e* (pAB03 vector with $\mathrm{P}_{\text {ermE* }}$ promoter instead of actII-ORF4/ $\mathrm{P}_{\text {actI }}$ activator/promoter system). pAB03e* oxyD was constructed by cloning $o x y D$ gene, excised from pAB03oxyD [1] with NdeI and $X b a I$ and ligated into pAB03e*. pAB03e*oxyDP was constructed by cloning oxyP gene, excised from pAB03oxyDP [1] with $X b a \mathrm{I}$, into $X b a \mathrm{I}$ site of pAB03e* oxyD downstream of $o x y D$ gene. pAB03e*oxyDPchdR was constructed by cloning chdR gene, excised from pAB03e"chdR with $\mathrm{ClaI}$ and HindIII (overhangs were filled in with Klenow polymerase), into $\mathrm{XbaI}$ (overhangs were filled in with Klenow polymerase) site of pAB03e" oxyDP downstream of $\operatorname{oxy} P$ gene.

\section{Heterologous expression of CHD BGC}

Cosmids carrying the CHD BGC, pOJ456CHD12, pOJ436CHD12, pOJ436e"chdRCHD12, pOJ436 "oxyDPCHD12 and pOJ436e* oxyDPchdRCHD12, and empty control cosmids, pOJ456, pOJ436, pOJ436e"chdR, pOJ436e"oxyDP and $\mathrm{pO} 4436 \mathrm{e}^{*}$ oxyDPchdR were transformed into E. coli ET12567 [30] carrying PUZ8002, which was then used as donor strain for intergeneric conjugation with $S$. albus del14. MS plates supplemented with $10 \mathrm{mM} \mathrm{MgCl}$ were overlaid with Apr and nalidixic acid after overnight incubation. Each exconjugant was further repatched onto MS agar containing Apr $(50 \mu \mathrm{g} / \mathrm{mL})$ and nalidixic acid $(25 \mu \mathrm{g} /$ $\mathrm{mL}$ ), followed by cultivation in liquid media as described above. Culture broths were extracted and analysed by liquid chromatography-mass spectroscopy (LC-MS) to check for production of CHD or CHD analogues.

\section{Liquid chromatography-mass spectroscopy analysis}

To measure the yield of CHD and 2-carboxamido2-deacetyl-chelocardin (CDCHD), S. albus culture broths were acidified to $\mathrm{pH} 1-2$ with $50 \%$ TFA, followed by extraction with $2 \mathrm{~V}$ of $\mathrm{MeOH}$. The extract was centrifuged and analyzed by LC-MS. All measurements were performed on a Dionex Ultimate 3000 LC system using a Luna C-18 [2] HST, $100 \times 2.0 \mathrm{~mm}, 2.5 \mu \mathrm{m}$ column (Phenomenex). Separation of $1 \mu \mathrm{l}$ sample was achieved by a linear gradient from (A) $\mathrm{H}_{2} \mathrm{O}+0.1 \%$ FA to (B) $\mathrm{ACN}+0.1 \% \mathrm{FA}$ at a flow rate of $500 \mu \mathrm{l} / \mathrm{min}$ 
and $45{ }^{\circ} \mathrm{C}$. The gradient was initiated by a $0.5 \mathrm{~min}$ isocratic step at $5 \% \mathrm{~B}$, followed by an increase to $95 \% \mathrm{~B}$ in 9 min to end up with a 1.5 min step at $95 \%$ B before reequilibration with initial conditions. To achieve better separation of peaks, gradient was extended to $18 \mathrm{~min}$. UV spectra were recorded by a DAD in the range from 200 to $600 \mathrm{~nm}$. The MS measurement was carried on an amaZon speed mass spectrometer (Bruker Daltonics, Bremen) using the standard ESI source. Mass spectra were acquired in centroid mode ranging from 200$2000 \mathrm{~m} / \mathrm{z}$ in positive ionization mode.

\section{Supplementary Information}

The online version contains supplementary material available at https://doi. org/10.1186/s12934-020-01495-x.

Additional file 1. Table S1. Bacterial strains and plasmids used in this study ${ }^{a}$. Table S2. Sequences of the oligonucleotide primers for PCR experiments used in this study. Table S3. Overexpression of SARPs otcR and chdB in $A$. sulphurea WT strain. Figure S1. Protein alignment of ChdB with closest homologs present in BGC encoding type II PKS. Close homologs of Streptomyces antibiotic regulatory protein (SARP) from oxytetracycline, SF2575, dactylocycline and chlorotetracycline BGC, OtcR, SsfT1, DacT1, $\mathrm{CtcB}$, respectively are presented. Gray colour denotes the similarity of the conserved amino acid residues. The OmpR/PhoB-type DNA-binding domain with a typical fold of the helix-turn-helix is marked with --, whereas the conserved DNA-binding sites are marked with *. Additional DNA-binding domain marked with $\sim$ contains three tetratricopeptide repeats (TPRs) and two C-terminal helices. The TPR motif generates a righthanded helical structure with an amphipathic channel that is thought to accommodate an alpha-helix of a target protein. Figure S2. Protein alignment of ChdC with closest homologs present in BGC encoding type II PKS. Close homologs from oxytetracycline, dactylocycline and chlorotetracycline BGC, OtcG, DacT3 and CtcA, respectively are presented. Gray colour denotes the similarity of the conserved amino acid residues. The Sigma-70 domain involved in binding to the -35 promoter element via a helix-turnhelix motif is marked with $\sim$, the signal receiver domain is marked ---, the TTA codon is marked with red rectangle, the phosphorylation site is marked with + , and the dimerization interface is marked with ${ }^{* * *}$. (7)

\section{Acknowledgements}

The authors wish to thank Acies Bio d.o.o. (Ljubljana, Slovenia) and Dr Andriy Luzhetskyy (AMEG group at Helmholtz-Institute for Pharmaceutical Research Saarland) for providing material for the research. We also thank Dr. Chantal Bader for technical assistance when preparing graphs presenting LC/MS analysis.

\section{Authors' contributions}

Conceptualization, TL, HP and RM; Methodology, TL, HP and RM; Validation, TL, SP, NZ and RM; Investigation, TL, SP and NZ; Resources, HP and RM; Data curation, TL, SP, and NZ Writing — original draft preparation, TL, SP, NZ, HP and RM; Supervision, TL, HP and RM; Project Administration, TL, HP and RM; Funding acquisition, HP, RM. All authors read and approved the final manuscript.

\section{Funding}

Open Access funding enabled and organized by Projekt DEAL. The study was supported in part by the Ministry of Higher Education, Science and Technology (Slovenian Research Agency, ARRS, grants no. J4-8226, P4-0116) to H.P. and SPIRIT Slovenija (grant no. P-MR-09/104) to T.L. Research in the R.M. laboratory is funded by the German Research Society (DFG) and the German Ministry of Education and Research (BMBF), while this study was also supported by the German Centre for Infection Research (DZIF) grant no. TTU09.814 and TTU09.821.

\section{Availability of data and materials}

All data generated or analysed during this study are included in this published article and its additional information files. We will introduce corrections into the current CHD gene cluster nucleotide sequence into GenBank Accession Number KC870000 once paper is at the final stages of reviewing.

\section{Ethics approval and consent to participate}

Not applicable.

\section{Consent for publication}

Not applicable.

\section{Competing interests}

The authors declare that they have no competing interests.

\section{Author details}

${ }^{1}$ Department of Microbial Natural Products, Helmholtz Institute for Pharmaceutical Research Saarland (HIPS)-Helmholtz Centre for Infection Research(HZI), and Department of Pharmacy, Saarland University Campus, Campus E8.1, 66123 Saarbrücken, Germany. ${ }^{2}$ German Center for Infection Research (DZIF), Partner Site Hannover-Braunschweig, 38124 Braunschweig, Germany. ${ }^{3}$ Department of Food Science and Technology, Biotechnical Faculty, University of Ljubljana, Jamnikarjeva 101, 1000 Ljubljana, Slovenia. ${ }^{4}$ Present Address: National Institute of Biology, Večna pot 111, 1000 Ljubljana, Slovenia.

Received: 28 August 2020 Accepted: 5 December 2020

Published online: 19 December 2020

\section{References}

1. Lukezic T, Lesnik U, Podgorsek A, Horvat J, PolakT, Sala M, et al. Identification of the chelocardin biosynthetic gene cluster from Amycolatopsis sulphurea: a platform for producing novel tetracycline antibiotics. Microbiology. 2013;159(Pt 12):2524-32.

2. Petković H, Lukežič T, Šušković J. Biosynthesis of oxytetracycline by Streptomyces rimosus. Past, present and future directions in the development of tetracycline antibiotics. Food Technol Biotechnol. 2017;55(1):3-13.

3. Hertweck C, Luzhetskyy A, Rebets Y, Bechthold A. Type II polyketide synthases: gaining a deeper insight into enzymatic teamwork. Nat Prod Rep. 2007;24(1):162-90.

4. Rasmussen B, Noller HF, Daubresse G, Oliva B, Misulovin Z, Rothstein DM, et al. Molecular basis of tetracycline action. Antimicrob Agents Chemother. 1991;35(11):2306-11.

5. Stepanek JJ, Lukežič T, Teichert I, Petković H, Bandow JE. Dual mechanism of action of the atypical tetracycline chelocardin. Biochim Biophys Acta. 2016;1864(6):645-54.

6. Molnar V, Matkovic Z, Tambic T, Kozma C. Klinicko-farmakolosko ispitivanje kelokardina u bolesnika s infekcijom mokracnih puta. Lijec Vjestn. 1977;99:560-2.

7. Proctor R, Craig W, Kunin C. Cetocycline, tetracycline analog. Antimicrob Agents Chemother. 1978;13(4):598-604.

8. Lesnik U, Lukezic T, Podgorsek A, Horvat J, PolakT, Sala M, et al. Construction of a new class of tetracycline lead structures with potent antibacterial activity through biosynthetic engineering. Angew Chem Int Ed Engl. 2015;54(13):3937-40.

9. Lukežič T, Fayad AA, Bader C, Harmrolfs K, Bartuli J, Groß S, et al. Engineering atypical tetracycline formation in Amycolatopsis sulphurea for the production of modified chelocardin antibiotics. ACS Chem Biol. 2019;14(3):468-77.

10. Grandclaudon C, Birudukota NVS, Elgaher WAM, Jumde RP, Yahiaoui S, Arisetti $\mathrm{N}$, et al. Semisynthesis and biological evaluation of amidochelocardin derivatives as broad-spectrum antibiotics. Eur J Med Chem. 2020;188:112005

11. Myronovskyi M, Rosenkränzer B, Nadmid S, Pujic P, Normand P, Luzhetskyy A. Generation of a cluster-free Streptomyces albus chassis strains for improved heterologous expression of secondary metabolite clusters. Metab Eng. 2018;49:316-24.

12. Pickens $L B$, Tang Y. Oxytetracycline biosynthesis. J Biol Chem. 2010;285(36):27509-15. 
13. Lešnik U, Gormand A, Magdevska V, Fujs Š, Raspor P, Hunter I, et al. Regulatory elements in tetracycline-encoding gene clusters: the otcG gene positively regulates the production of oxytetracycline in Streptomyces rimosus. Food Technol Biotechnol. 2009;47(3):323-30.

14. Yin S, Wang W, Wang $X$, Zhu Y, Jia X, Li S, et al. Identification of a clustersituated activator of oxytetracycline biosynthesis and manipulation of its expression for improved oxytetracycline production in Streptomyces rimosus. Microb Cell Fact. 2015;14(1):1-12.

15. Revill WP, Bibb MJ, Hopwood DA. Purification of a malonyltransferase from Streptomyces coelicolor A3(2) and analysis of its genetic determinant. J Bacteriol. 1995;177(14):3946-52.

16. Zhang W, Watanabe K, Wang CC, Tang Y. Investigation of early tailoring reactions in the oxytetracycline biosynthetic pathway. J Biol Chem. 2007;282(35):25717-25.

17. Menendez N, Nur-e-Alam M, Brana AF, Rohr J, Salas JA, Mendez C. Biosynthesis of the antitumor chromomycin A3 in Streptomycesgriseus. Chem Biol. 2004;11(1):21-32.

18. Pickens LB, Kim W, Wang P, Zhou H, Watanabe K, Gomi S, et al. Biochemical analysis of the biosynthetic pathway of an anticancer tetracycline SF2575. J Am Chem Soc. 2009;131(48):17677-89.

19. Xu W, Raetz LB, Wang P, Tang Y. An ATP-dependent ligase catalyzes the fourth ring cyclization in tetracycline biosynthesis. Tetrahedron. 2016;72(25):3599-604.

20. Zhang Z, Pan HX, Tang GL. New insights into bacterial type II polyketide biosynthesis. F1000Research. 2017;6(2016235):172.

21. Wang P, Zhang W, Zhan J, Tang Y. Identification of OxyE as an ancillary oxygenase during tetracycline biosynthesis. ChemBioChem. 2009;10(9):1544-50.

22. Wang P, Bashiri G, Gao X, Sawaya MR, Tang Y. Uncovering the enzymes that catalyze the final steps in oxytetracycline biosynthesis. J Am Chem Soc. 2013;135(19):7138-41.

23. Mitscher LA, Juvarkar JV, Rosenbrook W Jr, Andres WW, Schenk J, Egan RS. Structure of chelocardin, a novel tetracycline antibiotic. J Am Chem Soc. 1970;92(20):6070-1.

24. Chopra I. Tetracycline analogs whose primary target is not the bacterial ribosome. Antimicrob Agents Chemother. 1994;38(4):637-40.

25. Gramajo HC, Takano E, Bibb MJ. Stationary-phase production of the antibiotic actinorhodin in Streptomyces coelicolor A3(2) is transcriptionally regulated. Mol Microbiol. 1993;7(6):837-45.

26. Hennessen F, Miethke M, Zaburannyi N, Loose M, Schmiedel J, Fritzenwanker $M$, et al. Amidochelocardin overcomes resistance mechanisms exerted on tetracyclines and natural chelocardin. Antibiotics. 2020;9:619.
27. Oliva B, Gordon G, McNicholas P, Ellestad G, Chopra I. Evidence that tetracycline analogs whose primary target is not the bacterial ribosome cause lysis of Escherichia coli. Antimicrob Agents Chemother. 1992;36(5):913-9.

28. Rhodes PM, Hunter IS, Friend EJ, Warren M. Recombinant DNA methods for the oxytetracycline producer Streptomyces rimosus. Biochem Soc Trans. 1984;12(4):586-7.

29. Sambrook JF, Russell D. Molecular cloning: a laboratory manual (3-volume set), vol. 1. Cold Springs Harbour: Cold Springs Harbour Press; 2001.

30. MacNeil DJ, Gewain KM, Ruby CL, Dezeny G, Gibbons PH, MacNeil T. Analysis of Streptomyces avermitilis genes required for avermectin biosynthesis utilizing a novel integration vector. Gene. 1992;111(1):61-8.

31. Paget MS, Chamberlin L, Atrih A, Foster SJ, Buttner MJ. Evidence that the extracytoplasmic function sigma factor sigmaE is required for normal cell wall structure in Streptomyces coelicolor A3(2). J Bacteriol. 1999;181(1):204-11.

32. Kieser T, Bibb MJ, Buttner MJ, Chater KF, Hopwood DA. Practical streptomyces genetics. Society for general Microbiology. The John Innes Foundation 2000.

33. Bilyk O, Sekurova ON, Zotchev SB, Luzhetskyy A. Cloning and heterologous expression of the grecocycline biosynthetic gene cluster. PLoS ONE. 2016;11(7):e0158682.

34. Bilyk B, Horbal L, Luzhetskyy A. Chromosomal position effect influences the heterologous expression of genes and biosynthetic gene clusters in Streptomyces albus J1074. Microb Cell Fact. 2017;16(1):5.

35. Koshla O, Lopatniuk M, Rokytskyy I, Yushchuk O, Dacyuk Y, Fedorenko V, et al. Properties of Streptomyces albus $\mathrm{J} 1074$ mutant deficient in tRNALeuUAA gene bldA. Arch Microbiol. 2017;199(8):1175-83.

36. Madon J, Hutter R. Transformation system for Amycolatopsis (Nocardia) mediterranei Direct transformation of mycelium with plasmid DNA. J Bacteriol. 1991;173(20):6325-31.

37. Simpson JT, Wong K, Jackman SD, Schein JE, Jones SJM, Birol I. ABySS. Genome Res. 2009;19(6):1117-23.

38. Bierman M, Logan R, O'Brien K, Seno ET, Rao RN, Schoner BE. Plasmid cloning vectors for the conjugal transfer of DNA from Escherichia coli to Streptomyces spp. Gene. 1992;116(1):43-9.

\section{Publisher's Note}

Springer Nature remains neutral with regard to jurisdictional claims in published maps and institutional affiliations.
Ready to submit your research? Choose BMC and benefit from:

- fast, convenient online submission

- thorough peer review by experienced researchers in your field

- rapid publication on acceptance

- support for research data, including large and complex data types

- gold Open Access which fosters wider collaboration and increased citations

- maximum visibility for your research: over 100M website views per year

At BMC, research is always in progress.

Learn more biomedcentral.com/submissions 